\title{
Stigmatization and the Predictors of Reported Discrimination among HIV Positive Patients Receiving Care in Hospitals in Imo State, Nigeria
}

Chukwuma B Duru' ${ }^{1}$, Anthony C Iwu ${ }^{2 *}$, Kevin C Diwe ${ }^{1}$, Uche R Oluoha ${ }^{2}$, Irene A Merenu ${ }^{1}$, Chinyere M. Aguocha ${ }^{3}$, Ugochukwu C Madubueze ${ }^{4}$, Nera P Kadiri-Eneh ${ }^{5}$, Emmanuel U Ndukwu' ${ }^{2}$, Ikechi Ohale ${ }^{2}$ and Ernest Nwaigbo ${ }^{2}$

${ }^{1}$ Department of Community Medicine, Imo State University, Owerri, Imo State, Nigeria

${ }^{2}$ Department of Community Medicine, Imo State University Teaching Hospital, Orlu Imo State, Nigeria

${ }^{3}$ Department of Psychiatry, Imo State University, Owerri, Imo State, Nigeria.

${ }^{4}$ Department of Community Medicine, Federal Teaching Hospital, Abakaliki, Nigeria.

${ }^{5}$ Department of Community Medicine, University of Port Harcourt Teaching Hospital, Rivers State, Nigeria

\section{Abstract}

Background: HIV related Stigma and discrimination is a reflection of fear of the unknown, driven by ignorance and helplessness that results in negative attitude, beliefs and unfair treatment towards those living with HIV.

Objective: To assess knowledge of stigmatization, prevalence of reported discrimination and the socio-demographic and care predictors of discrimination among HIV positive patients receiving care in Health Care Institutions in Imo State, Nigeria.

Methods: A cross sectional analytical design using a simple random sampling technique to select 422 HIV positive patients attending the adult HIV clinics from two public health care institutions in Imo State. Data was collected using a pretested semi structured questionnaire. Descriptive analyses were done with frequencies and summary statistics. Chi square statistics were computed to determine significant relationships and simple binary logistic regression was used to determine predictors of HIV related discrimination. The $p$-value was set at 0.05 significance level.

Results: The mean age of the respondents was $37 \pm 6.3$ with a male to female ratio of 1:1.7. The overall level of knowledge of stigmatization and discrimination was poor in more than half of the respondents $(55.2 \%)$. The prevalence of stigmatization and reported discrimination was $33.2 \%$ of which, close to two thirds of it occurred among the females $(62.9 \%)$. The main source of discrimination was from family and relatives $(45.0 \%)$ with close to half of the respondents having suicidal thoughts after experiencing discrimination (46.4\%). The predictors of HIV related discrimination and stigmatization reported in this study were; being within the ages of 26-35 years, (OR 2.16), having at least one or more children (OR: 3.72), residing in their community of origin (OR: 2.00), residing in Orlu (OR: 3.77) or Okigwe (OR: 3.29) zones of Imo State, receiving advice to do the HIV test from a health personnel (OR: 2.52) and doing a HIV test and receiving a positive result from a private hospital (OR:2.22) or health centre (OR: 2.11).

Conclusion: Stigmatization and discrimination of people living with HIVIAIDS are created by individuals and communities influenced by socio-demographic and care factors which continues to perpetuate the transmission of HIV; and therefore, it remains an important issue of public health concern that must be addressed.

Keywords: Stigmatization; Discrimination; Prevalence; Predictors; HIV patients; Hospitals; Nigeria

\section{Introduction}

Stigma refers to a negative or unfavourable perception that embodies the individual or community's attitude or beliefs; or public policies towards self or a group of people with certain characteristics that fall short of society's expectations. It is a reflection of fear and the unknown, driven by ignorance and helplessness. As a consequence of stigma, or when stigma is acted upon, discrimination is said to occur, which is an unfair act or unjust treatment towards an individual or group based on identified characteristics [1,2].

Stigma and discrimination is of public health concern as it can negatively or adversely affect the physical health, mental health and overall wellbeing of the individual and community as a whole [1]. HIV/ AIDS remains a stigmatizing disease and people living with HIV/AIDS (PLWHA) are very vulnerable to stigmatization and discrimination due to the persisting myths, misinformation and false beliefs that always associate HIV/AIDS with death [3]. This is, in spite of the significant progress that has been made in the treatment of the disease since its discovery.

Stigma, discrimination and HIV appear to have an unyielding cyclical relationship; with vulnerable people being discriminated, marginalized and subsequently becoming susceptible to, or infected with HIV/AIDS. These people, who become infected with HIV are further stigmatized and discriminated upon, therefore, perpetuating this cycle of negative perception and actions. This continues to create barriers or discriminatory acts throughout the lives of people living with HIV with respect to their family, work and health care. These barriers created, are obviously seen, felt and reportable; and this constitute their personal experiences of discrimination (reported discrimination). These barriers occur especially, when it involves rejection by friends and families, denial of access to health care or employment, or restrictions of travel $[1,3,4]$.

*Corresponding author: Anthony C Iwu, Department of Community Medicine, Imo State University Teaching Hospital, Orlu Imo State, Nigeria; Tel: 2348033483968 ; E-mail: iwuchinedu@yahoo.com

Received November 04, 2017; Accepted November 13, 2017; Published November 20, 2017

Citation: Duru CB, Iwu AC, Diwe KC, Oluoha UR, Merenu IA, et al. (2017) Stigmatization and the Predictors of Reported Discrimination among HIV Positive Patients Receiving Care in Hospitals in Imo State, Nigeria. J AIDS Clin Res 8: 745 doi: 10.4172/2155-6113.1000745

Copyright: (C) 2017 Duru CB, et al. This is an open-access article distributed under the terms of the Creative Commons Attribution License, which permits unrestricted use, distribution, and reproduction in any medium, provided the original author and source are credited. 
According to UNAIDS, more than $50 \%$ of people in more than one third of countries with available data, reported having unfavourable attitudes and beliefs towards people living with HIV/AIDS; with about one in every eight persons living with HIV/AIDS not having access to healthcare services as a consequence [4,5]. This was highlighted in a previous study which observed that, PLWHA who report high levels of stigma, were more likely to report poor access to healthcare [6].

HIV related stigmatization and discrimination not only influences social, economic, physical and psychological wellbeing of the individual but it undermines public health interventions targeted towards preventing and controlling the spread of HIV infections. This hinders HIV counselling, testing and treatment uptake, status disclosure and the opportunities to adopt safer sexual behavioural practices [7-9]. Furthermore, there are increased challenges to public health interventions in countries that enact laws that criminalise or prosecute PLWHA for non-disclosure, exposure and transmission [10]. These laws deter HIV testing and disclosure and therefore, conceals the actual burden of HIV. This, consequently shifts the strategy of HIV prevention from interventions that includes public health strategies to interventions that constitute solely, the preventive practices of individual partners of HIV infected persons [11].

The people most at risk or infected with HIV continue to face stigma and discrimination based on socio-demographic and other factors not limited to age, race, socioeconomic status, sex, sexual orientation, geographic location, health care settings and perceived or actual health status $[4,10]$

Women and girls are most affected by stigmatization and discrimination as they are at an increased risk of social rejection, losing shelter and their children, and also lacking the ability to survive with the attendant consequences of depression and suicide $[3,9,12]$. Also, women in marital relationships especially women with older husbands and in lower socioeconomic households were significantly more likely to experience stigma and discrimination from family and friends [13].

Similarly, people infected with HIV who are engaged with work, are also at an increased risk of stigmatization and discrimination from co-employees and employers, where on disclosure, are either isolated or employment terminated; and for those currently unemployed, are denied opportunities of work and as a consequence, lose their source of income and livelihood. In 2012, it was reported in Nigeria that $45 \%$ and $27 \%$ of people living with HIV had either lost their source of income or were refused job opportunities respectively on account of their status [14].

Also, HIV-related discrimination in healthcare is an important issue especially in developing countries where HIV testing can be done without consent or appropriate counselling, or where health care providers minimize contact, isolate patient, deny treatment or increase the payment, before services are rendered; or even violate their privacy and confidentiality [4].

However, HIV related stigmatization and discrimination cannot be comprehensively and effectively tackled without an understanding of the level of relevant knowledge and the underlying factors associated with discrimination. Thus, the aim of this study is to assess the level of knowledge of stigmatization and the socio-demographic and care factors associated with discrimination among HIV positive patients receiving care in Imo State, Nigeria.

\section{Methodology}

\section{Study area}

The study was conducted within the adult HIV clinics of Imo State University Teaching Hospital, Orlu (IMSUTH) situated in Orlu Local Government Area (LGA) and Imo State Specialist Hospital, Umuguma situated in Owerri West LGA in Imo State, Nigeria. Orlu LGA is one of the 12 LGAs that comprise Imo West Senatorial zone and occupies an area of $132.9 \mathrm{~km}^{2}$ with a population density of about 1,074 persons per $\mathrm{km}^{2}$ [15]. Owerri West LGA is one of the 9 LGAs that comprise Imo East Senatorial zone and occupies an area of $297 \mathrm{~km}^{2}$ with a population density of about 343 persons per $\mathrm{km}^{2}$. [16].

\section{Study population/study design/selection criteria}

The study population comprised adult HIV infected patients accessing antiretroviral therapy from the HIV clinics of Imo State University Teaching Hospital and Imo State Specialist Hospital, Umuguma. The study design was a cross sectional hospital based descriptive survey. The inclusion criteria for enrolment into the survey after informed consent was any adult HIV patient attending clinics in the selected hospitals and who were clinically stable as at the time of conduct of study. Those just recently diagnosed or on their first visit to the clinic as at the time of survey were excluded.

\section{Sample size estimation}

The minimum sample size was calculated using the Cochrane formula for populations greater than 10,000 [15].

$$
\mathrm{n}=\frac{\mathrm{Z}^{2} \mathrm{pq}}{\mathrm{d}^{2}}
$$

When $\mathrm{n}=$ minimum sample size, $\mathrm{Z}=$ Standard normal deviate corresponding to $5 \%$ significant level,

$\mathrm{p}=$ proportion of people living with HIV virus that experienced discrimination on account of their status was 45\% [14], $\mathrm{q}=1-0.45$, $\mathrm{d}=$ tolerable error of margin set at $0.05 . \mathrm{Z}=1.96, \mathrm{p}=0.45, \mathrm{q}=0.55, \mathrm{n}=380$. The sample size used for the survey while taking into account nonresponses was 422 .

\section{Sampling technique}

A simple random sampling technique was used to select the respondents that were studied. Equal number of patients attending HIV clinics (211 individuals) was selected and studied from each health facility. From the registry of patients for clinic appointments for each clinic day, about 12 and 18 patients were randomly selected by balloting based on population size from Imo State University Teaching Hospital and Imo State Specialist Hospital respectively until the required sample size was obtained. Those selected that were not eligible were replaced by next eligible person.

\section{Data Collection and Analysis}

Data was collected using a pretested, semi structured, interviewer administered questionnaire during a 6 -week period from $18^{\text {th }}$ July to $29^{\text {th }}$ August 2016. The questionnaire was developed by the researchers and pretested in the HIV clinic of Holy Rosary Hospital Emekuku situated outside the study area. The content validity of the questionnaire was established qualitatively by assessing each question against the intended construct.

The questionnaire comprised three sections; section one: the sociodemographic and care characteristics, section two: Awareness and 
Knowledge of stigmatization, section three: Prevalence and Pattern of discrimination.

The level of knowledge of stigmatization was determined by scoring the questions that assessed knowledge. For a single response question, an appropriate answer was scored 2; an inappropriate answer was scored 0 . For a multiple response question, up to 2 appropriate answers was scored 1; from 3 to 5 appropriate answers was scored 3 and greater than 5 appropriate answers was scored 5 . The aggregate scores for each respondent's level of knowledge of stigmatization was translated to a percentage and assessed against a scale of less than $60 \%$ for poor and $60 \%$ or more for good.

Data was cleaned and validated manually, and analysed using Software Package for Social Sciences (IBM-SPSS) version 22. Descriptive statistics (frequency tables and summary indices) were generated. Chi Square was used to test association between HIV related discrimination and the socio-demographic and care factors. A simple regression model was developed with the socio-demographic and care factors that had significant associations and binary logistic regression was applied to determine the predictors of HIV related discrimination. $P$ value was set at 0.05 significance level.

\section{Results}

Four hundred and twenty two copies of the questionnaire were distributed and all the copies were completely and correctly filled with a response rate of $100 \%$.

\section{Socio-demographic and care characteristics of HIV positive respondents receiving care in Imo State}

Most of the respondents were of the Igbo tribe (87.7\%). The mean age of the respondents was $37.1 \pm 6.3$ years with close to two thirds of the respondents' being female (63.5\%), more than two fifths were single (43.3\%) and majority, belonging to either the Pentecostal or Catholic faith (81.8\%). Close to one third of the respondents were traders $(30.8 \%)$ with a majority having at most, a secondary level of education (64.7\%). More than half of the respondents earned a monthly income of less than 20,000 naira (56.6\%), resided in Owerri zone (60.0\%) and not in their communities of origin (55.7\%). More than two fifths of the respondents had no children $(41.5 \%)$ but close to two thirds, had more than three occupants in the household $(60.7 \%)$ with a majority of the respondents living with family members $(72.0 \%)$. Close to half of the respondents received advice to do the HIV test from a health personnel (48.8\%) with most of the respondents receiving their positive HIV diagnosis from a public hospital (79.6\%) and more than half undergoing pre-test counselling sessions $(54.7 \%)$ and greater proportion of the respondents (67.3\%) enrolled and receiving HIV care and treatment for more than 6 months (Table 1).

\section{Awareness and knowledge of stigmatization among HIV pos- itive respondents receiving care in Imo State}

A majority of the respondents were aware of what stigmatization is $(69.0 \%)$ with their major source of information being from radio broadcast (67.7\%). Majority of the respondents aware of stigmatization were of the understanding that stigmatization is either anything that brings disgrace $(67.0 \%)$ or anything that sets you apart (60.5\%). Though all the respondents labelled HIV as a stigmatizing disease, only about one third of the respondents labelled leprosy as a stigmatizing disease (34.7\%). Though a majority of the respondents reported knowing the consequences of stigmatization $(76.6 \%)$, only about one eights of those that reported knowing, indicated dismissal from work (12.6\%)

\begin{tabular}{|c|c|c|}
\hline Variable & Category & $\begin{array}{c}\text { Frequency }(\%) \\
n=422\end{array}$ \\
\hline Age (years) & $\begin{array}{l}18-25 \\
26-35\end{array}$ & $\begin{array}{r}69(16.4) \\
157(37.2)\end{array}$ \\
\hline Mean age $(37.1 \pm 6.3)$ & $\begin{array}{c}36-45 \\
>45\end{array}$ & $\begin{array}{l}126(29.9) \\
70(16.6)\end{array}$ \\
\hline Gender & $\begin{array}{l}\text { Male } \\
\text { Female }\end{array}$ & $\begin{array}{l}154(36.5) \\
268(63.5)\end{array}$ \\
\hline Religion & $\begin{array}{l}\text { Pentecostal } \\
\text { Catholic } \\
\text { Orthodox } \\
\text { Others }^{1}\end{array}$ & $\begin{array}{c}186(44.1) \\
159(37.7) \\
59(14.0) \\
18(4.3)\end{array}$ \\
\hline Marital status & $\begin{array}{l}\text { Single } \\
\text { Married } \\
\text { Others }^{2}\end{array}$ & $\begin{array}{l}183(43.4) \\
177(41.9) \\
62(14.7)\end{array}$ \\
\hline Educational level & $\begin{array}{c}\text { Tertiary } \\
\text { Secondary } \\
\text { None/Primary }\end{array}$ & $\begin{array}{c}149(35.3) \\
213(50.5) \\
60(14.2)\end{array}$ \\
\hline Ethnicity & $\begin{array}{l}\text { Igbo } \\
\text { Non-lgbo }\end{array}$ & $\begin{array}{l}370(87.7) \\
52(12.3)\end{array}$ \\
\hline Occupation & $\begin{array}{c}\text { Trader } \\
\text { Student } \\
\text { Civil servant } \\
\text { Artisan } \\
\text { Unemployed } \\
\text { Others }^{3}\end{array}$ & $\begin{array}{c}130(30.8) \\
67(15.9) \\
62(14.7) \\
56(13.3) \\
41(9.7) \\
66(15.6)\end{array}$ \\
\hline $\begin{array}{l}\text { Monthly income (Naira) } \\
(\mathrm{N} 20,000=\$ 55)\end{array}$ & $\begin{array}{c}\leq 20,000 \\
>20,000 \\
\text { Can't assess }\end{array}$ & $\begin{array}{c}239(56.6) \\
142(33.6) \\
41(9.7)\end{array}$ \\
\hline Number of children & $\begin{array}{c}\text { None } \\
1-3 \\
>3\end{array}$ & $\begin{array}{l}175(41.5) \\
146(34.6) \\
101(23.9)\end{array}$ \\
\hline Number in household & $\begin{array}{l}\leq 3 \\
>3\end{array}$ & $\begin{array}{l}166(39.3) \\
256(60.7)\end{array}$ \\
\hline Household living companion & $\begin{array}{c}\text { Family member } \\
\text { Alone } \\
\text { Others }{ }^{4}\end{array}$ & $\begin{array}{c}304(72.0) \\
53(12.6) \\
65(15.4)\end{array}$ \\
\hline Residing in your community of origin & $\begin{array}{l}\text { Yes } \\
\text { No }\end{array}$ & $\begin{array}{l}187(44.3) \\
235(55.7)\end{array}$ \\
\hline Place of residence & $\begin{array}{l}\text { Owerri zone } \\
\text { Orlu zone } \\
\text { Okigwe zone } \\
\text { Outside Imo }\end{array}$ & $\begin{array}{c}253(60.0) \\
88(20.9) \\
58(13.7) \\
23(5.5)\end{array}$ \\
\hline Place of HIV diagnosis & $\begin{array}{c}\text { Public Hospital } \\
\text { Private hospital/Lab } \\
\text { Health centre }\end{array}$ & $\begin{array}{c}336(79.6) \\
54(12.8) \\
32(7.6)\end{array}$ \\
\hline Duration of HIV care & $\begin{array}{l}\leq 6 \text { months } \\
>6-36 \text { months } \\
>36 \text { months }\end{array}$ & $\begin{array}{l}138(32.7) \\
164(38.9) \\
120(28.4)\end{array}$ \\
\hline Source of advice to do HIV test & $\begin{array}{l}\text { Health personnel } \\
\text { Self } \\
\text { Relations/friends } \\
\text { Others }{ }^{5}\end{array}$ & $\begin{array}{c}206(48.8) \\
89(21.1) \\
41(9.7) \\
86(20.4)\end{array}$ \\
\hline Pre-test counselling & $\begin{array}{l}\text { Yes } \\
\text { No }\end{array}$ & $\begin{array}{l}231(54.7) \\
191(45.3)\end{array}$ \\
\hline
\end{tabular}

1-Muslim, pagan, 2-separated, divorced, living with partner, 3-farmer, teacher professional, 4-friends, strangers, 5-parents, antenatal co-patients

Table 1: Socio-demographic and care characteristics of HIV positive respondents receiving care in Imo State.

and general neglect (13.0\%) as consequences. Similarly, even though most of the respondents reported that stigmatization poses challenges to them $(96.9 \%)$, only $6.4 \%$ and $17.7 \%$ see erosion of human rights and receiving poor health care respectively as challenges faced due to stigmatization (Table 2).

A majority of the respondents had a poor level of knowledge about stigmatization (55.2\%) (Figure 1). 
Citation: Duru CB, Iwu AC, Diwe KC, Oluoha UR, Merenu IA, et al. (2017) Stigmatization and the Predictors of Reported Discrimination among HIV Positive Patients Receiving Care in Hospitals in Imo State, Nigeria. J AIDS Clin Res 8: 745. doi: 10.4172/2155-6113.1000745

Page 4 of 8

\section{Prevalence and pattern of reported discrimination among HIV positive respondents receiving care in Imo State}

About one third of the respondents reported experiencing any

\begin{tabular}{|c|c|c|}
\hline Variable & Category & Frequency (\%) \\
\hline $\begin{array}{l}\text { Are you aware of what } \\
\text { stigmatization is? }(n=422)\end{array}$ & $\begin{array}{l}\text { Yes } \\
\text { No }\end{array}$ & $\begin{array}{l}291(69.0) \\
131(31.0)\end{array}$ \\
\hline $\begin{array}{l}\text { *Sources of information } \\
(n=291)\end{array}$ & $\begin{array}{c}\text { Radio } \\
\text { Television } \\
\text { Health personnel } \\
\text { Seminar/workshop } \\
\text { Books } \\
\text { Church } \\
\text { Friends/relatives } \\
\text { Newspaper }\end{array}$ & $\begin{array}{l}197(67.7) \\
114(39.2) \\
111(38.1) \\
31(10.7) \\
24(8.2) \\
16(5.5) \\
11(3.8) \\
11(3.8)\end{array}$ \\
\hline $\begin{array}{l}\text { *What is your understanding of } \\
\text { stigmatization? }(n=291)\end{array}$ & $\begin{array}{c}\text { Anything that brings disgrace } \\
\text { Anything that sets you apart } \\
\text { Facing criticism from society } \\
\text { Anything that makes you feel } \\
\text { guilty } \\
\text { Denial of human right }\end{array}$ & $\begin{array}{l}195(67.0) \\
176(60.5) \\
145(49.8) \\
78(26.8) \\
74(25.4)\end{array}$ \\
\hline $\begin{array}{l}\text { *What diseases are } \\
\text { stigmatizing? }(n=291)\end{array}$ & $\begin{array}{c}\text { HIV } \\
\text { Ebola } \\
\text { Tuberculosis } \\
\text { Leprosy } \\
\text { Chicken pox } \\
\text { Measles } \\
\text { Lassa fever }\end{array}$ & $\begin{array}{c}291(100) \\
275(94.5) \\
267(91.8) \\
101(34.7) \\
20(6.9) \\
9(3.1) \\
7(2.4)\end{array}$ \\
\hline $\begin{array}{l}\text { Do you know the consequences } \\
\text { of stigmatization? }(n=291)\end{array}$ & $\begin{array}{l}\text { Yes } \\
\text { No }\end{array}$ & $\begin{array}{l}223(76.6) \\
68(23.4)\end{array}$ \\
\hline $\begin{array}{l}\text { *What are the consequences of } \\
\text { stigmatization }(n=223)\end{array}$ & $\begin{array}{c}\text { Social abuse } \\
\text { Emotional abuse } \\
\text { Sexual denial by partner } \\
\text { Physical abuse } \\
\text { General neglect } \\
\text { Dismiss from work } \\
\text { Restricted from public place } \\
\text { Financial denial }\end{array}$ & $\begin{array}{c}161(72.2) \\
141(63.2) \\
88(39.5) \\
33(14.8) \\
29(13.0) \\
28(12.6) \\
9(4.0) \\
7(3.1)\end{array}$ \\
\hline $\begin{array}{l}\text { Does stigmatization pose } \\
\text { challenges for people with HIV? } \\
(n=291)\end{array}$ & $\begin{array}{l}\text { Yes } \\
\text { No }\end{array}$ & $\begin{array}{l}282(96.9) \\
9(3.1)\end{array}$ \\
\hline $\begin{array}{l}\text { *What are the challenges faced? } \\
(n=282)\end{array}$ & $\begin{array}{c}\text { Neglect by family } \\
\text { Non-acceptance by peers and } \\
\text { community } \\
\text { Psychosocial damage } \\
\text { Receiving poor health care/ } \\
\text { education } \\
\text { Erosion of human right }\end{array}$ & $\begin{array}{c}164(58.2) \\
121(42.9) \\
92(32.6) \\
50(17.7) \\
18(6.4)\end{array}$ \\
\hline
\end{tabular}

*multiple responses

Table 2: Awareness and knowledge of stigmatization among HIV positive respondents receiving care in Imo state.

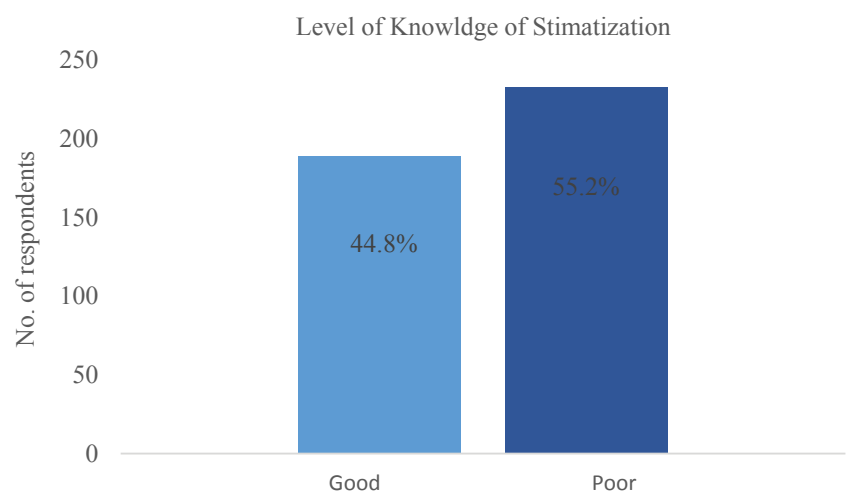

Figure 1: Level of knowledge of stigmatization among HIV positive respondents receiving Care in Imo State. form of discrimination (33.2\%) and the sources of discrimination were mainly from family members and relatives $(45.0 \%)$ or spouse and partner $(30.7 \%)$. The major type of discrimination experienced was either sexual denial from partner $(37.1 \%)$ or social abuse by family/friends (36.4\%) which was frequently experienced, in close to half of the respondents (49.3\%). A majority of the respondents after experiencing discrimination felt either suicidal $(46.4 \%)$ or had an indescribable feeling (24.3\%). Less than half of the respondents reported having additional health problems due to discrimination (45.7\%) and of those that reported being discriminated, developing self-determination $(45.7 \%)$ or counselling by friends $(37.9 \%)$ were the main forms of management employed to handle the feeling of discrimination (Table 3 ).

Of those that were discriminated, close to two thirds were female HIV positive patients (62.9\%). Though, there was a similar female to male ratio among those discriminated compared to those not discriminated (Figure 2).

\section{Socio demographic and care factors associated with discrimi- nation among HIV positive respondents receiving care in Imo State}

The following socio-demographic and care factors were significantly associated with HIV related discrimination; Age $(\mathrm{p}=0.002)$, religion $(\mathrm{p}<0.0001)$, occupation $(\mathrm{p}=0.017)$, number of children $(\mathrm{p}<0.0001)$, residing in your community of origin $(\mathrm{p}=0.001)$, place of residence $(\mathrm{p}<0.0001)$, place of HIV diagnosis $(\mathrm{p}=0.006)$, duration of HIV

\begin{tabular}{|c|c|c|}
\hline Variable & Category & $\begin{array}{l}\text { Frequency } \\
\quad(\%)\end{array}$ \\
\hline $\begin{array}{l}\text { Have you ever experienced } \\
\text { discrimination? }(n=422)\end{array}$ & $\begin{array}{l}\text { Yes } \\
\text { No }\end{array}$ & $\begin{array}{l}140(33.2) \\
282(66.8)\end{array}$ \\
\hline *Sources of discrimination $(n=140)$ & $\begin{array}{c}\text { Family/Relatives } \\
\text { Spouse/Partner } \\
\text { Health workers } \\
\text { Colleagues } \\
\text { Friends }\end{array}$ & $\begin{array}{l}63(45.0) \\
43(30.7) \\
31(22.1) \\
22(15.7) \\
15(10.7)\end{array}$ \\
\hline *Type of discrimination? $(n=140)$ & $\begin{array}{c}\text { Sexual denial by partner } \\
\text { Social abuse by family/ } \\
\text { friends } \\
\text { Restricted from public } \\
\text { function } \\
\text { Financial neglect by } \\
\text { family } \\
\text { Physical abuse by family } \\
\text { Dismissed from work } \\
\text { Emotional abuse by } \\
\text { colleagues }\end{array}$ & $\begin{array}{l}52(37.1) \\
51(36.4) \\
19(13.6) \\
17(12.1) \\
14(10.0) \\
10(7.1) \\
7(5.0)\end{array}$ \\
\hline $\begin{array}{l}\text { How often are you discriminated? } \\
(n=140)\end{array}$ & $\begin{array}{l}\text { Frequently } \\
\text { Occasionally } \\
\text { Sometimes }\end{array}$ & $\begin{array}{l}69(49.3) \\
29(20.7) \\
42(30.0)\end{array}$ \\
\hline $\begin{array}{l}\text { How do you feel after being } \\
\text { discriminated? }(n=140)\end{array}$ & $\begin{array}{c}\text { Suicidal } \\
\text { Indescribable } \\
\text { Low esteem } \\
\text { Guilty } \\
\text { Ashamed }\end{array}$ & $\begin{array}{c}65(46.4) \\
34(24.3) \\
22(15.7) \\
10(7.1) \\
9(6.4)\end{array}$ \\
\hline $\begin{array}{l}\text { Has it caused you any additional health } \\
\text { problems? }(n=140)\end{array}$ & $\begin{array}{l}\text { Yes } \\
\text { No }\end{array}$ & $\begin{array}{l}64(45.7) \\
76(54.3)\end{array}$ \\
\hline $\begin{array}{l}\text { *How do you manage the feeling of being } \\
\text { discriminated? }(n=140)\end{array}$ & $\begin{array}{l}\text { Self determination } \\
\text { Counselled by friends } \\
\text { Counselled by partner } \\
\text { Inspiration from media/ } \\
\text { books } \\
\text { Counselled by family } \\
\text { Counselled by clergy }\end{array}$ & $\begin{array}{l}64(45.7) \\
53(37.9) \\
31(22.1) \\
31(22.1) \\
29(20.7) \\
18(12.9)\end{array}$ \\
\hline
\end{tabular}

*multiple responses

Table 3: Prevalence and pattern of reported discrimination among HIV positive respondents receiving care in Imo state. 
Citation: Duru CB, Iwu AC, Diwe KC, Oluoha UR, Merenu IA, et al. (2017) Stigmatization and the Predictors of Reported Discrimination among HIV Positive Patients Receiving Care in Hospitals in Imo State, Nigeria. J AIDS Clin Res 8: 745. doi: 10.4172/2155-6113.1000745

Page 5 of 8

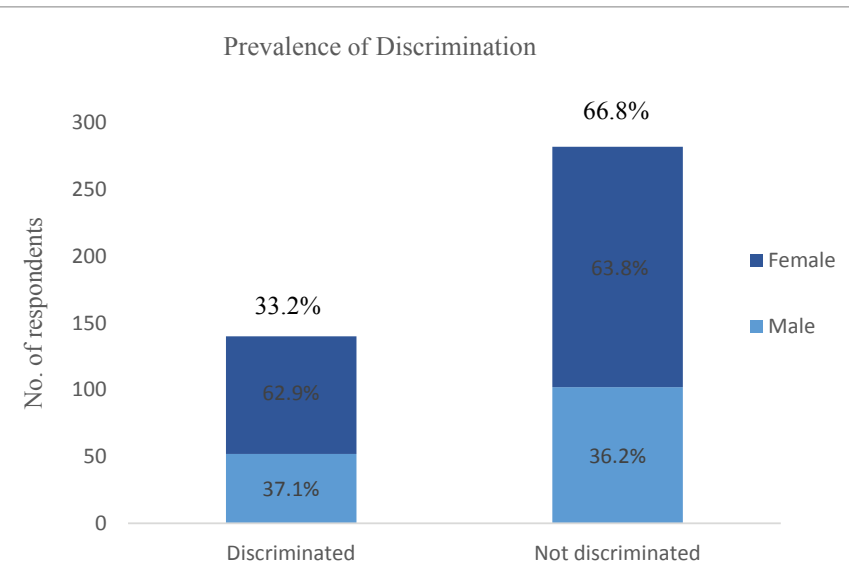

Figure 2: Prevalence of reported discrimination according to gender and among HIV Positive respondents receiving care in Imo State.

\begin{tabular}{|c|c|c|c|c|c|c|}
\hline Variable & $\begin{array}{c}\text { Discriminated } \\
(\%)\end{array}$ & $\begin{array}{c}\text { Not } \\
\text { Discriminated } \\
(\%)\end{array}$ & Total (\%) & $x^{2}$ & df & p-value \\
\hline $\begin{array}{l}\text { Age (years) } \\
18-25 \\
26-35 \\
36-45 \\
>45 \\
\text { Total }\end{array}$ & $\begin{array}{c}19(27.5) \\
70(44.6) \\
32(25.4) \\
19(27.1) \\
140(33.2)\end{array}$ & $\begin{array}{c}50(72.5) \\
87(55.4) \\
94(74.6) \\
51(72.9) \\
282(66.8)\end{array}$ & $\begin{array}{c}69(100) \\
157(100) \\
126(100) \\
70(100) \\
422(100)\end{array}$ & 14.80 & 3 & $0.002^{*}$ \\
\hline $\begin{array}{l}\text { Gender } \\
\text { Male } \\
\text { Female } \\
\text { Total }\end{array}$ & $\begin{array}{c}52(33.8) \\
88(32.8) \\
140(33.2)\end{array}$ & $\begin{array}{l}102(66.2) \\
180(67.2) \\
282(66.8)\end{array}$ & $\begin{array}{l}154(100) \\
268(100) \\
422(100)\end{array}$ & 0.04 & 1 & 0.845 \\
\hline $\begin{array}{l}\text { Religion } \\
\text { Pentecostal } \\
\text { Catholic } \\
\text { Orthodox } \\
\text { Others } \\
\text { Total } \\
\text { Marital Status } \\
\text { Single } \\
\text { Married } \\
\text { Others } \\
\text { Total }\end{array}$ & $\begin{array}{c}45(24.2) \\
67(42.1) \\
26(44.1) \\
2(11.1) \\
140(33.2) \\
53(29.0) \\
65(36.7) \\
22(35.5) \\
140(33.2)\end{array}$ & $\begin{array}{c}141(75.8) \\
92(57.9) \\
33(55.9) \\
16(88.9) \\
282(66.8) \\
130(71.0) \\
112(63.3) \\
40(64.5) \\
282(66.8)\end{array}$ & $\begin{array}{c}186(100) \\
159(100) \\
59(100) \\
18(100) \\
422(100) \\
\\
183(100) \\
177(100) \\
62(100) \\
422(100)\end{array}$ & 19.64 & 2 & $0.000^{*}$ \\
\hline $\begin{array}{l}\text { Educational } \\
\text { level } \\
\text { Tertiary } \\
\text { Secondary } \\
\text { None/Primary } \\
\text { Total }\end{array}$ & $\begin{array}{c}45(30.2) \\
73(34.3) \\
22(36.7) \\
140(33.2)\end{array}$ & $\begin{array}{c}104(69.8) \\
140(65.7) \\
38(63.3) \\
282(66.8)\end{array}$ & $\begin{array}{c}149(100) \\
213(100) \\
60(100) \\
422(100)\end{array}$ & 1.04 & 2 & 0.595 \\
\hline $\begin{array}{l}\text { Ethnicity } \\
\text { Igbo } \\
\text { Non-Igbo } \\
\text { Total }\end{array}$ & $\begin{array}{c}126(34.1) \\
14(26.9) \\
140(33.2)\end{array}$ & $\begin{array}{c}244(65.9) \\
38(73.1) \\
282(66.8)\end{array}$ & $\begin{array}{c}370(100) \\
52(100) \\
422(100)\end{array}$ & 1.05 & 1 & 0.306 \\
\hline $\begin{array}{l}\text { Occupation } \\
\text { Trading } \\
\text { Student } \\
\text { Civil servant } \\
\text { Artisan } \\
\text { Unemployed } \\
\text { Others } \\
\text { Total }\end{array}$ & $\begin{array}{c}51(39.2) \\
26(38.8) \\
10(16.1) \\
22(39.3) \\
14(34.1) \\
17(25.8) \\
140(33.2)\end{array}$ & $\begin{array}{c}79(60.8) \\
41(61.2) \\
52(83.9) \\
34(60.7) \\
27(65.9) \\
49(74.2) \\
282(66.8)\end{array}$ & $\begin{array}{c}130(100) \\
67(100) \\
62(100) \\
56(100) \\
41(100) \\
66(100) \\
422(100)\end{array}$ & 13.83 & 5 & $0.017^{*}$ \\
\hline $\begin{array}{l}\text { Monthly } \\
\text { Income } \\
\leq 20,000 \\
>20,000 \\
\text { Can't assess } \\
\text { Total }\end{array}$ & $\begin{array}{c}80(33.5) \\
44(31.0) \\
16(39.0) \\
140(33.2)\end{array}$ & $\begin{array}{c}159(66.5) \\
98(69.0) \\
25(61.0) \\
282(66.8)\end{array}$ & $\begin{array}{c}239(100) \\
142(100) \\
41(100) \\
422(100)\end{array}$ & 0.95 & 2 & 0.622 \\
\hline
\end{tabular}

\begin{tabular}{|c|c|c|c|c|c|c|}
\hline $\begin{array}{l}\text { No. of Children } \\
\text { None } \\
1-3 \\
>3 \\
\text { Total }\end{array}$ & $\begin{array}{c}34(19.4) \\
69(47.3) \\
37(36.6) \\
140(33.2)\end{array}$ & $\begin{array}{c}141(80.6) \\
77(52.7) \\
64(63.4) \\
282(66.8)\end{array}$ & $\begin{array}{l}175(100) \\
146(100) \\
101(100) \\
422(100)\end{array}$ & 28.53 & 2 & $0.000^{*}$ \\
\hline $\begin{array}{l}\text { Number in } \\
\text { Household } \\
\leq 3 \\
>3 \\
\text { Total }\end{array}$ & $\begin{array}{c}60(36.1) \\
80(31.3) \\
140(33.2)\end{array}$ & $\begin{array}{l}106(63.9) \\
176(68.8) \\
282(66.8)\end{array}$ & $\begin{array}{l}166(100) \\
256(100) \\
422(100)\end{array}$ & 1.09 & 1 & 0.297 \\
\hline $\begin{array}{l}\text { Household } \\
\text { living } \\
\text { companion } \\
\text { Family member } \\
\text { Alone } \\
\text { Others } \\
\text { Total }\end{array}$ & $\begin{array}{c}104(34.2) \\
14(26.4) \\
22(33.8) \\
140(33.2)\end{array}$ & $\begin{array}{c}200(65.8) \\
39(73.6) \\
43(66.2) \\
282(66.8)\end{array}$ & $\begin{array}{c}304(100) \\
53(100) \\
65(100) \\
422(100)\end{array}$ & 1.25 & 2 & 0.535 \\
\hline $\begin{array}{l}\text { Living in your } \\
\text { community of } \\
\text { origin } \\
\text { Yes } \\
\text { No } \\
\text { Total }\end{array}$ & $\begin{array}{c}78(41.7) \\
62(26.4) \\
140(33.2)\end{array}$ & $\begin{array}{l}109(58.3) \\
173(73.6) \\
282(66.8)\end{array}$ & $\begin{array}{l}187(100) \\
235(100) \\
422(100)\end{array}$ & 11.04 & 1 & $0.001^{*}$ \\
\hline $\begin{array}{l}\text { Place of } \\
\text { residence } \\
\text { Owerri zone } \\
\text { Orlu zone } \\
\text { Okigwe zone } \\
\text { Outside Imo } \\
\text { Total }\end{array}$ & $\begin{array}{c}59(23.3) \\
47(53.4) \\
29(50.0) \\
5(21.7) \\
140(33.2)\end{array}$ & $\begin{array}{l}194(76.7) \\
41(46.6) \\
29(50.0) \\
18(78.3) \\
282(66.8)\end{array}$ & $\begin{array}{c}253(100) \\
88(100) \\
58(100) \\
23(100) \\
422(100)\end{array}$ & 36.10 & 3 & $0.000^{*}$ \\
\hline $\begin{array}{l}\text { Place of HIV } \\
\text { diagnosis } \\
\text { Public Hospital } \\
\text { Private hospital/ } \\
\text { Lab } \\
\text { Health centre } \\
\text { Total }\end{array}$ & $\begin{array}{c}99(29.5) \\
26(48.1) \\
15(46.9) \\
140(33.2)\end{array}$ & $\begin{array}{c}237(70.5) \\
28(51.9) \\
17(53.1) \\
282(66.8)\end{array}$ & $\begin{array}{c}336(100) \\
54(100) \\
32(100) \\
422(100)\end{array}$ & 10.26 & 2 & $0.006^{*}$ \\
\hline $\begin{array}{l}\text { Duration of HIV } \\
\text { care } \\
\leq 6 \text { months } \\
>6-36 \text { months } \\
>36 \text { months } \\
\text { Total }\end{array}$ & $\begin{array}{c}52(37.7) \\
71(43.3) \\
17(14.2) \\
140(33.2)\end{array}$ & $\begin{array}{c}86(62.3) \\
93(56.7) \\
103(85.8) \\
282(66.8)\end{array}$ & $\begin{array}{l}138(100) \\
164(100) \\
120(100) \\
422(100)\end{array}$ & 28.39 & 2 & $0.000^{*}$ \\
\hline $\begin{array}{l}\text { Source of } \\
\text { advice to do } \\
\text { HIV test } \\
\text { Health } \\
\text { personnel } \\
\text { Self } \\
\text { Relations/friends } \\
\text { Others } \\
\text { Total } \\
\end{array}$ & $\begin{array}{l}87(42.2) \\
20(22.5) \\
11(26.8) \\
22(25.6) \\
140(33.2)\end{array}$ & $\begin{array}{l}119(57.8) \\
69(77.5) \\
30(73.2) \\
64(74.4) \\
282(66.8)\end{array}$ & $\begin{array}{c}206(100) \\
89(100) \\
41(100) \\
86(100) \\
422(100)\end{array}$ & 15.20 & 3 & $0.002^{*}$ \\
\hline $\begin{array}{l}\text { Pre-test } \\
\text { counselling } \\
\text { Yes } \\
\text { No } \\
\text { Total }\end{array}$ & $\begin{array}{c}74(32.0) \\
66(34.6) \\
140(33.2)\end{array}$ & $\begin{array}{l}157(68.0) \\
125(65.4) \\
282(66.8)\end{array}$ & $\begin{array}{l}231(100) \\
191(100) \\
422(100)\end{array}$ & 0.30 & 1 & 0.584 \\
\hline
\end{tabular}

\section{*significant}

Table 4: Socio demographic and care factors associated with discrimination among HIV positive respondents receiving care in Imo State.

treatment and care $(\mathrm{p}<0.0001)$ and source of advice to do HIV test $(\mathrm{p}=0.002)$ (Table 4).

Predictors of discrimination among HIV positive respondents receiving care in Imo State

Respondents between the ages of 26-35 years were significantly more likely to have reported discrimination when compared to those aged more than 45 years (OR: 2.16; 1.169-3.989, $\mathrm{p}=0.013$ ). Respondents 
that practice Pentecostal Christian religion and other religion (Islam and paganism) were significantly less likely to have reported discrimination when compared to the Catholic Christian religion (OR: 0.44; 0.277 $0.694, \mathrm{p}<0.0001)$ and (OR: 0.17; 0.038-0.772, $\mathrm{p}=0.011)$ respectively.

Respondents, who are civil servants were significantly less likely to have reported discrimination when compared to those that were unemployed (OR: 0.37; 0.146-0.945, $\mathrm{p}=0.034$ ).

Respondents with 1-3 children and more than 3 children were significantly more likely to have reported discrimination when compared to those without children (OR: 3.72; 2.264-6.101, $\mathrm{p}<0.0001$ ) and (OR: $2.40 ; 1.381-4.162, \mathrm{p}=0.002$ ) respectively.

Respondents residing in their community of origin were significantly more likely to have reported discrimination when compared to those residing outside their community of origin (OR: 2.00; 1.324-3.011, $\mathrm{p}=0.001)$.

Respondents residing in Orlu and Okigwe zones were significantly more likely to have reported discrimination when compared to those residing in Owerri zone (OR: 3.77; 2.263-6.279, $\mathrm{p}<0.0001)$ and $(\mathrm{OR}$ : $3.29 ; 1.820-5.941, \mathrm{p}<0.0001)$ respectively.

Respondents advised by a health personnel to do a HIV test were significantly more likely to have reported discrimination when compared to those that advised themselves to do a HIV test (OR: 2.52; 1.427-4.457, $\mathrm{p}=0.001$ ).

Respondents, who received a positive diagnosis of HIV from private hospital/laboratory and from a health centre were significantly more likely to have reported discrimination when compared to those that received their positive diagnosis from a public hospital (OR: 2.22; 1.241-3.983, $\mathrm{p}=0.006)$ and (OR: $2.11 ; 1.015-4.396, \mathrm{p}=0.042)$ respectively.

Respondents receiving treatment and care for more than 36 months were significantly less likely to have reported discrimination when compared to those that received treatment and care for 6 months or less (OR: 0.27; 0.147-0.506, $\mathrm{p}<0.0001$ ) (Table 5).

\section{Discussion}

This study assessed knowledge of stigmatization, prevalence of reported discrimination and the socio-demographic and care predictors of discrimination among HIV positive patients receiving care in Imo, Nigeria.

The level of knowledge of stigmatization among a majority of the respondents was low and probably could explain why the level of reported experience of discrimination was also low; because without the awareness or knowledge of what constitutes stigmatization, the complete experience of being discriminated including human rights violations may not be fully appreciated. Discrimination may have been obvious or significant to the respondents, only when they were emotionally or socially neglected by their partners, family and friends on account of their HIV status; as other aspects, such as HIV testing without consent, violations of their privacy and confidentiality may not have been considered as forms of discrimination as observed in the present study. While more than one third of the respondents reported denial of sex from their partners and social abuse from family and friends as the forms of discrimination experienced, only less than one tenth reported dismissal from work or emotional abuse from colleagues. This appears not to be unusual, because it is only when you disclose your status to colleagues that the issue of discrimination if any, could arise from the work environment; and as such, this may explain

\begin{tabular}{|c|c|c|c|}
\hline Variable & $\begin{array}{c}\text { OR } \\
\text { (estimate) }\end{array}$ & $95 \%(\mathrm{Cl})$ & p-value \\
\hline $\begin{array}{l}\text { Age (years) } \\
18-25 \\
26-35 \\
36-45 \\
>45\end{array}$ & $\begin{array}{l}1.02 \\
2.16 \\
0.91 \\
1.00\end{array}$ & $\begin{array}{c}0.484-2.151 \\
1.169-3.989 \\
0.471-1.772 \\
-\end{array}$ & $\begin{array}{c}1.000 \\
\mathbf{0 . 0 1 3}^{*} \\
0.791 \\
-\end{array}$ \\
\hline $\begin{array}{l}\text { Religion } \\
\text { Pentecostal } \\
\text { Catholic } \\
\text { Orthodox } \\
\text { Others }\end{array}$ & $\begin{array}{l}0.44 \\
1.00 \\
1.08 \\
0.17\end{array}$ & $\begin{array}{c}0.277-0.694 \\
- \\
0.592-1.977 \\
0.038-0.772\end{array}$ & $\begin{array}{l}\mathbf{0 . 0 0 0 *} \\
- \\
0.791 \\
\mathbf{0 . 0 1 1}^{*} \\
-\end{array}$ \\
\hline $\begin{array}{l}\text { Occupation } \\
\text { Trading } \\
\text { Student } \\
\text { Civil servant } \\
\text { Artisan } \\
\text { Unemployed } \\
\text { Others }\end{array}$ & $\begin{array}{l}1.25 \\
1.22 \\
0.37 \\
1.25 \\
1.00 \\
0.67\end{array}$ & $\begin{array}{c}0.597-2.597 \\
0.543-2.753 \\
0.146-0.945 \\
0.539-2.881 \\
- \\
0.286-1.564\end{array}$ & $\begin{array}{c}0.560 \\
0.624 \\
\mathbf{0 . 0 3 4 ^ { \star }} \\
0.603 \\
- \\
0.354\end{array}$ \\
\hline $\begin{array}{l}\text { No. of Children } \\
\text { None } \\
1-3 \\
>3\end{array}$ & $\begin{array}{l}1.00 \\
3.72 \\
2.40\end{array}$ & $\begin{array}{c}- \\
2.264-6.101 \\
1.381-4.162\end{array}$ & $\begin{array}{c}- \\
0.000^{*} \\
0.002^{*}\end{array}$ \\
\hline $\begin{array}{l}\text { Residing in your } \\
\text { community of origin } \\
\text { Yes } \\
\text { No }\end{array}$ & $\begin{array}{l}2.00 \\
1.00\end{array}$ & $\begin{array}{c}1.324-3.011 \\
-\end{array}$ & $\begin{array}{c}0.001^{*} \\
-\end{array}$ \\
\hline $\begin{array}{l}\text { Place of residence } \\
\text { Owerri zone } \\
\text { Orlu zone } \\
\text { Okigwe zone } \\
\text { Outside Imo }\end{array}$ & $\begin{array}{l}1.00 \\
3.77 \\
3.29 \\
0.91\end{array}$ & $\begin{array}{c}- \\
2.263-6.279 \\
1.820-5.941 \\
0.325-2.566\end{array}$ & $\begin{array}{c}- \\
\mathbf{0 . 0 0 0 ^ { * }} \\
\mathbf{0 . 0 0 0 ^ { * }} \\
0.862\end{array}$ \\
\hline $\begin{array}{l}\text { Place of HIV diagnosis } \\
\text { Public Hospital } \\
\text { Private hospital/Lab } \\
\text { Health centre }\end{array}$ & $\begin{array}{l}1.00 \\
2.22 \\
2.11\end{array}$ & $\begin{array}{c}- \\
1.241-3.983 \\
1.015-4.396\end{array}$ & $\begin{array}{c}- \\
0.006^{\star} \\
0.042^{*}\end{array}$ \\
\hline $\begin{array}{l}\text { Duration of HIV care } \\
\leq 6 \text { months } \\
>6-36 \text { months } \\
>36 \text { months }\end{array}$ & $\begin{array}{l}1.00 \\
1.26 \\
0.27\end{array}$ & $\begin{array}{c}- \\
0.795-2.005 \\
0.147-0.506\end{array}$ & $\begin{array}{c}- \\
0.322 \\
\mathbf{0 . 0 0 0 *}\end{array}$ \\
\hline $\begin{array}{l}\text { Source of advice to do } \\
\text { HIV test } \\
\text { Health personnel } \\
\text { Self } \\
\text { Relations/friends } \\
\text { Others }\end{array}$ & $\begin{array}{l}2.52 \\
1.00 \\
1.27 \\
1.19\end{array}$ & $\begin{array}{c}1.427-4.457 \\
- \\
0.540-2.964 \\
0.592-2.375\end{array}$ & 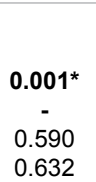 \\
\hline
\end{tabular}

Table 5: Predictors of discrimination among HIV Positive respondents receiving care in Imo State.

why very few of the respondents reported work dismissal or emotional abuse from colleagues as the forms of discrimination experienced. According to Iwu et al. [7], a study done in Imo State among HIV positive male patients on sero-status disclosure revealed that, none of the respondents were willing to disclose their status to their work colleague for fear of discrimination.

This level of discrimination in the present study was similarly observed in studies done in Cameroon [17] and Madagascar [18] where $26 \%$ and $36 \%$ of the patients reported having been victims of discrimination respectively. The level of education and the socioeconomic status of the respondents, may also have contributed to their level of knowledge of stigmatization and hence, could have influenced what was regarded as discrimination by the respondents. As observed in the present study, close to two thirds of the respondents' highest level of education attained, was a secondary education with a majority of the respondents either unemployed or engaged as traders or artisans earning monthly incomes of less than 20,000 Naira (\$55). Though, in the present study, it was observed that the level of education and monthly income was not significantly associated with the 
respondents' experience of discrimination and also, with respect to the level of education, this lack of association was similarly observed in a previous African study [17]. On the contrary, some other studies [19,20] reported that, low levels of education among respondents, appear to be protective against perceived stigma among people living with HIV. This also, was not consistent with a study done among HIV positive African-Americans, where it was observed that higher education was associated with low levels of perceived stigma [21]. Perceived stigma is characterised by personalized experiences of real or imagined fear of societal attitudes and a concern that discriminatory acts could occur. This, invariably influences the report of discrimination; as people who experience the burden of perceived stigma, are more likely to report discrimination [21-23].

According to Tenkorang et al. [24], stigmatization and discrimination assumes a social class dimension; hence, being socioeconomically disadvantaged i.e. poor, uneducated and unemployed, create the opportunities and further perpetuate the occurrences of HIV stigmatization and discrimination.

Unlike gender and marital status; age, religion, occupation and parity were observed in the present study to be associated with reported discrimination. Respondents within the ages of 26-35 years and having at least a child were more likely than those above 45 years and without a child respectively to report discrimination; and respondents who are civil servants and of the Pentecostal Christian faith were significantly less likely than those who were unemployed and of the Catholic Christian faith respectively to have reported discrimination. The mandatory HIV counselling and testing during antenatal care for pregnant women and the inevitable disclosure of the woman's HIV status, and by extension, the speculative HIV status of the partner, subsequently increases the likelihood for discrimination. This may be a possible explanation for why the respondents with at least a child were more likely to report discrimination.

The Catholic faith is known to have a more conservative stance on social issues and therefore may appear to have HIV related discriminatory tendencies and as such, this may explain to some extent why those of the Pentecostal faith were less likely to have experienced discrimination. Also, with respect to occupation, even though it is not unusual in our environment for a mandatory HIV test before employment, it is quite unusual for employees in the public sector to be mandated to do a HIV test, and this, coupled with the unlikelihood of HIV status disclosure, may be an explanation why the respondents who are employed as civil servants with undisclosed HIV status were less likely to have reported discrimination. Furthermore, though the present study observed a similar lack of association between gender and discrimination with a previous study in Cameroon [17], the relationship between age and discrimination was not consistently observed.

In the present study, place of residence and living in your community of origin was significantly associated with the report of discrimination. It was observed that those that live in Okigwe and Orlu zones were more likely than Owerri zone to report discrimination. Okigwe and Orlu zones can be categorized as mainly comprising rural and semiurban areas while Owerri zone where the State capital is located, can be categorized as mainly comprising urban and semi-urban areas. According to Alemu et al. [25], PLWHA in resource limited settings who reside in rural areas appear to have a higher level of stigma and discrimination. This may be associated with the level of ignorance, social exposure and the greater attachments to certain traditions, customs and beliefs that result in heightened personal and community fear [26]. Also, communal familiarity and cohabitation with family and extended family members increases the likelihood for HIV status disclosure, dissemination and subsequent discrimination. This, may possibly explain why respondents residing in their community of origin were significantly more likely than those residing outside to have reported discrimination.

The perpetuation of stigmatizing attitudes and discrimination by healthcare workers has been widely reported, ranging from issues of confidentiality, verbal harassment to denial of treatment [4,27-29]. As a result, it is not unexpected that respondents in the present study who came in contact with the healthcare system and were advised by health personnel to do a HIV test were significantly more likely to have reported discrimination than those who advised themselves. Furthermore, there has been an increased support in HIV care management and support facilities by the Government and donor agencies mainly towards public health care institutions and this has been associated with increased opportunities for education and training on HIV/AIDS. Also, it is not surprising that respondents, who received a positive diagnosis of HIV from private hospitals were significantly more likely than those who received their positive diagnosis from public hospitals to have reported discrimination. Probably, this associated increase in the opportunities for education and training on HIV/AIDS may have contributed to some extent, in reducing the ignorance and fear usually associated with HIV. This consequently, may have influenced the stigmatizing attitudes and discrimination by the healthcare workers in public health care institutions in relation to the private health care institutions. Though, on the contrary, a study among health workers in Northern Nigeria reported that, there was no statistically significant difference in stigmatizing attitudes towards PLWHA between the public and private health care workers; and in spite of education and training, there was no reduction in discriminatory attitudes among the health care workers [30,31]. Finally, the longer PLWHA are in care and living healthy lives, the more accommodating their family and friends are, as they begin to realize and appreciate that HIV is neither an automatic death sentence nor is it transmissible by regular physical contact. As a consequence, stigmatizing attitudes and discrimination begin to decrease overtime and this may probably explain the observation in the present study, where the respondents that received care for more than 36 months were significantly less likely to have experienced discrimination.

\section{Conclusion}

Stigmatization and discrimination of PLWHA are created by individuals and communities and remains an important issue of public health concern, as it is influenced by a range of socio-demographic and care factors. This also, in turn, influences individual disclosure, treatment uptake, prevention and transmission of HIV. Therefore, interventions to prevent or reduce HIV-related stigma and discrimination must remain an integral component of the comprehensive approach to the management of HIV/AIDS. Hence, it is crucial to develop targeted interventions that take into cognizance socio-demographic and care factors, as this will improve significantly the health and quality of life of PLWHA. Furthermore, both the PLWHA and the healthcare institutions must be empowered through regular education and training, so that PLWHA will know and understand their rights and therefore be in a position to advocate, promote and protect their fundamental human rights in the context of HIV/AIDS; and the healthcare workers will be better equipped, especially psychologically to effectively manage and care for PLWHA.

\section{Acknowledgement}

We thank all the participants in this study, the research assistants who helped during the data collection and the patient support care staff. 
Citation: Duru CB, Iwu AC, Diwe KC, Oluoha UR, Merenu IA, et al. (2017) Stigmatization and the Predictors of Reported Discrimination among HIV Positive Patients Receiving Care in Hospitals in Imo State, Nigeria. J AIDS Clin Res 8: 745. doi: 10.4172/2155-6113.1000745

\section{Author's Contributions}

All the authors participated in the study.

\section{References}

1. Centre for Disease Control and Prevention (CDC) (2016) Stigma and Discrimination. National Center for HIVIAIDS, Viral Hepatitis, STD, and TB Prevention.

2. Harapan H, Feramuhawan S, Kurniawan H, Anwar S, Andalas M, et al. (2013) HIV-related stigma and discrimination: A study of health care workers in Banda Aceh, Indonesia. Med J Indones 22: 22-29.

3. AVERT (2017) Global information and education on HIV and AIDS: HIV Stigma and Discrimination.

4. http://www.unaids.org/sites/default/files/media_asset/2017ZeroDiscrimination HealthCare.pdf

5. http://www.unaids.org/sites/default/files/media_asset/WAD2015_report_en_ part01.pdf

6. Sayles JN, Wong MD, Kinsler JJ, Martins D, Cunningham WE, et al (2009) The association of stigma with self-reported access to medical care and antiretroviral therapy adherence in persons living with HIVIAIDS. J Gen Intern Med 24: 1101-1108.

7. Iwu AC, Duru CB, Diwe KC, Uwakwe KA, Merenu IA, et al. (2017) Sexua behaviour, sero-status disclosure and willingness to disclose status among HIV positive male patients receiving care in hospitals in Imo State, Nigeria. J AIDS Clin Res 8: 1-10.

8. http://www.unaids.org/sites/default/files/media_asset/2014unaidsguidancenote_ stigma_en.pdf

9. https://www.icrw.org/wp-content/uploads/2016/10/Common-at-its-Core-HIVRelated-Stigma-Across-Contexts.pdf

10. Bernard EJ, Cameron S (2016) Advancing HIV Justice 2: Building momentum in global advocacy against HIV criminalisation. HIV Justice Network and the Global Network of People Living with HIV.

11. http://files.unaids.org/en/media/unaids/contentassets/documents/document/ 2013/05/20130530 Guidance Ending_Criminalisation.pdf

12. http://www.thewellproject.org/hiv-information/stigma-and-discriminationagainst-women-living-hiv

13. Halli SS, Hussain Khan CG, Moses S, Blanchard J, Washington R, et al. (2017) Family and community level stigma and discrimination among women living with HIVIAIDS in a high HIV prevalence district of India. Journal of HIVIAIDS \& Social Services 16: 4-19.

14. http://www.ilo.org/wcmsp5/groups/public/---ed_protect/---protrav/---ilo_aids/ documents/publication/wcms_185808.pdf

15. http://www.scirp.org/(S(i43dyn45teexjx455qlt3d2q))/reference/ReferencesPapers. aspx?ReferencelD=1837525

16. National Population Commission (NPC) (2006) Federal Republic of Nigeria Population and Housing Census: Priority table volume III. NPC.
17. Essomba EN, Kollo B, Ngambi MK, Assomba L, Etang K, et al. (2014) Stigma and discrimination associated with HIVIAIDS in health care settings: A comparative study in two hospitals of different categories in Douala-Cameroon. J Med Biomed Sci 3: 14-22.

18. Andrianasolo RL, Rakotoarivelo RA, Randriarimanana D, Angijiro PG, Randria MJ (2011) Discrimination of HIV infected persons in medical settings in Madagascar. Med Mal Infect 41: 2-6.

19. Li Z, Sheng $Y$ (2014) Investigation of perceived stigma among people living with human immunodeficiency virus/acquired immune deficiency syndrome in Henan Province, China. Int J Nurs Sci 1: 385-388.

20. Sorsdahl KR, Mall S, Stein DJ, Joska JA (2011) The prevalence and predictors of stigma amongst people living with HIVIAIDS in the Western Province. AIDS Care 23: 680-685.

21. Galvan FH, Davis EM, Banks D, Bing EG (2008) HIV stigma and social support among African Americans. AIDS Patient Care STDS 22: 423-436.

22. Black BP, Miles MS (2002) Calculating the risks and benefits of disclosure in African American women who have HIV. J Obstet Gynecol Neonatal Nurs 31: 688-697.

23. Kaiser Family Foundation (2004) Survey of Americans on HIVIAIDS: Par three-experiences opinions by race/ethnicity, age. Kaiser Family Foundation, Washington, D.C.

24. Tenkorang EY, Owusu AY (2013) Examining HIV-related stigma and discrimination in Ghana: What are the major contributors? Sex Health 10: 253262.

25. Alemu T, Biadgilign S, Deribe K, Escudero HR (2013) Experience of stigma and discrimination and the implications for healthcare seeking behavior among people living with HIVIAIDS in resource-limited setting. SAHARA J 10: 1-7.

26. Heckman TG, Somlai AM, Kalichman SC, Franzoi SL, Kelly JA (1998) Psychosocial differences between urban and rural people living with HIVIAIDS. J Rural Health 14: 138-145.

27. Feyissa GT, Abebe L, Girma E, Woldie M (2012) Stigma and discrimination against people living with HIV by healthcare providers, Southwest Ethiopia. BMC Public Health 12: 522

28. Banteyerga H, Kidanu A, Abebe F (2005) Perceived Stigmatization and Discrimination by Healthcare Providers towards Persons with HIVIAIDS. USAIDS.

29. Ekstrand ML, Ramakrishna J, Bharat S, Heylen E (2013) Prevalence and drivers of HIV stigma among health providers in urban India: Implications for interventions. J Int AIDS Soc 16: 18717.

30. Amoran OE (2011) HIV related stigmatizing attitude and practice among health care workers in Northern Nigeria. J Infect Dis Immunity 3: 226-232.

31. Ezedinachi EN, Ross MW, Meremiku M, Essien EJ, Edem CB, et al. (2002) The impact of an intervention to change health workers' HIVIAIDS attitudes and knowledge in Nigeria: A controlled trial. Public Health 116: 106-112. 